\title{
Using Pareto Optimum to Choose Module's Computing PlatForms OF MOBILE ROBOT WiTH MODULAR ARCHITECTURE
}

\author{
Victor Andreev, Valerii Kim \& Pavel Pletenev
}
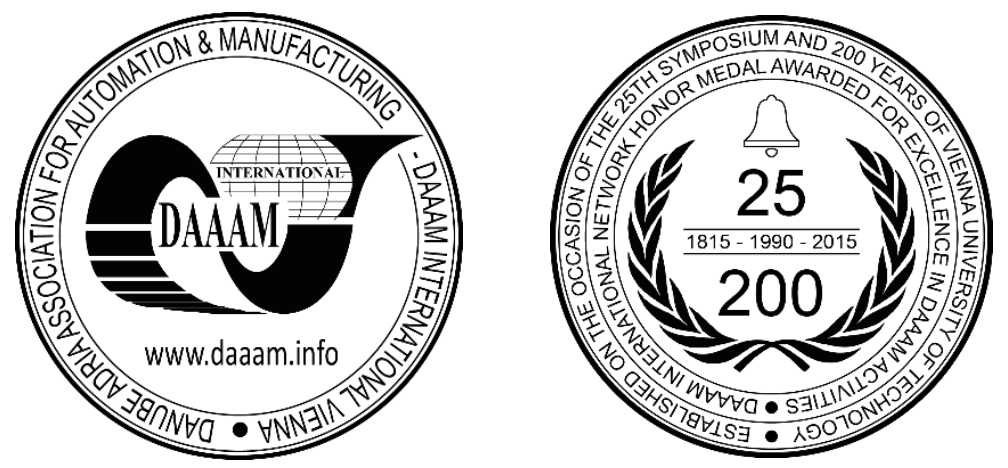

This Publication has to be referred as: Andreev, V[iktor]; Kim, V[alerii] \& Pletenev, P[avel] (2018). Using Pareto Optimum to Choose Module's Computing Platforms of Mobile Robot with Modular Architecture, Proceedings of the 29th DAAAM International Symposium, pp.0559-0565, B. Katalinic (Ed.), Published by DAAAM International, ISBN 978-3-902734-20-4, ISSN 1726-9679, Vienna, Austria

DOI: $10.2507 / 29$ th.daaam.proceedings.081

\begin{abstract}
This paper tries to address a sufficiently complex task - choice of module's computing platform for implementation of information-measuring and control system (IMCS) of full-functional modules of mobile robot with modular architecture. The full functionality of a mechatronic device is the ability to perform its goal function using only its own facilities for executing instructions from an external control system. This condition sets for robot's IMCS some specific requirements on selection of corresponding computational platform, which one could separate into objective, subjective and computative-objective criteria. This paper proposes use of discrete optimization with Pareto-optimum to select viable alternatives from computing platforms which can be both off-the-shelf and perspective products. One of the main criteria - such embedded computing platforms must have suitable computational power, low weight and sizes. The paper provides descriptions of different computing platforms, as well as the rationale for main quality criteria of them. An example of Pareto optimization for a specific IMCS solution for some types of mobile robot modules with modular architecture is provided.
\end{abstract}

Keywords: modular robot; mobile robot; pareto optimum; computing platform; discrete multicriteria optimization

\section{Introduction}

This paper is part of research towards developing a modular architecture for mobile robots with fully functional modules [1-3]. The full functionality of a mechatronic device is the ability to perform its goal function using only its own facilities for executing instructions from an external control system [1]. The main purpose of the work is to simplify and formalize one of the stages of module's design - the stage of choosing a prototype of the computational platform for the module. The module's computational platform (MCP) is an electronic computing device or a set of such devices that work together and implement the network interface of the module and its control system using the appropriate software. 
To do this, it is necessary to consider the currently available embedded computational platforms and their sets, then to determine the essential criteria for the quality of the MCP and to carry out appropriate optimization on their basis. The purpose of optimization is to significantly reduce the search space for alternative versions of the MCP.

The computing platform for control systems of robot modules must have, at the bare minimum, an embedded computing device capable of operating in an Ethernet network over UDP protocol with a performance sufficient to implement the functional purpose of the module. MCP should also have minimum weight and size parameters and the lowest possible cost [3]. Due to the distribution of calculations between the microprocessors of the modules, the control system of the modular robot as a whole has a higher performance compared to the control systems that are built on a single computer.

\section{State-of-art prototyping module's computational platform}

In order to select a computational platform for the robot module, on the basis of which it is possible to implement the mechanism of intermodule information interaction, an analytical review of various solutions was performed.

\subsection{Arduino Uno}

This module's computational platform [4] was first introduced in 2011. MCP is built on the Atmel AVR ATMega328P microcontroller running at $16 \mathrm{MHz}$ and has a memory of $32 \mathrm{~KB}$. MCP has 14 controlled I/O pins to communicate with external devices, 6 of which can generate an 8-bit PWM signal. The platform has 6 channels of analog-to-digital conversion (ADC). There is no interface to access the local area network on the Arduino Uno.

\subsection{Arduino Ethernet Shield}

This is an extension module for Arduino [5], which is installed in a standard connector of a Arduino-compatible MCP. Its implementation is based on W5100 chip with built-in buffer of $16 \mathrm{~KB}$. The chip implements the network (IP) Protocol stack and supports TCP and UDP protocols. Communication with the Arduino is via the serial peripheral interface(SPI).

The Board has a connector for MicroSD cards, which are also connected to the main board via SPI. The module uses 6 pins on Arduino connector. Its functionality is limited to the ability to receive or send data on only 4 simultaneous connections - this is a limitation of the W5100 chip, which is installed on the Ethernet Shield. The ZeroMQ implementation on the basis of this module, respectively, is severely limited in its functionality.

\subsection{Arduino Mega}

MCP [6] is built on the Atmel AVR ATMega2650 microcontroller. The microcontroller operates at a frequency of $16 \mathrm{MHz}$, has $256 \mathrm{~KB}$ of memory and has 54 general purpose I/O (GPIO) pins for interaction with external devices. 15 pins of this MCP can generate a hardware 8 bits and 16 bit PWM signal. Also the Arduino Mega has 16 channels of 10 bit ADC. There is no local area network (LAN) access interface on the Arduino Mega, but it can be added by installing the Ethernet Shield module.

\subsection{Arduino Due}

MCP [7] is based on ATMEL AT91SAM3X8E microcontroller with 32-bit data and address bus, using the ARM architecture. The microcontroller operates at a frequency of $84 \mathrm{MHz}$. Memory: $512 \mathrm{~KB}$ of ROM and 96 KB of RAM. The Arduino Due has 54 GPIO, 12 of which can generate a PWM signal. MCP has 12 channels of analog-to-digital conversion. The Board does not have its own interface to connection to the LAN, which can be created using the additional Ethernet Shield module.

\subsection{Taijiuino Due Pro R3 + Ethernet PHY}

MCP[8] is based on ATMEL AT91SAM3X8E microcontroller with 32-bit bus, created on ARM architecture. The microcontroller operates at a frequency of $84 \mathrm{MHz}$. Memory capacity: $512 \mathrm{~KB}$ of ROM and $96 \mathrm{~KB}$ of RAM. Like the Arduino Due, the MCP has 54 digital inputs / outputs, 12 of which can generate a PWM signal. MCP has 12 channels of analog-to-digital conversion. MCP Board additionally (compared to Arduino Due) has contacts for connecting a special chip that implements the physical layer of the OSI model for Ethernet.

The microcontroller AT91SAM3X8E has hardware implementing channel level of OSI (MAC) for 10/100 MB Ethernet. Thus, it is possible to implement the entire Ethernet standard on this microcontroller. The disadvantage of this board is its relatively complex to purchase - it must be ordered directly from the manufacturer from China. It should also be noted that the Ethernet library for this board is still in development, and the main developer has abandoned it. 


\subsection{Arduino Yun}

In MCP [9] two microcontrollers are used at once; they can be named "Executive" and "Control". One can call Atmel AVR ATmega32u4 an "Executive" microcontroller as it interacts with external devices, collects sensor readings and generates a hardware PWM signals. The microcontroller operates at a frequency of $16 \mathrm{MHz}$, has $32 \mathrm{~KB}$ of program memory and $2.5 \mathrm{~KB}$ of RAM. It communicates with external devices via 20 digital inputs / outputs, seven of which can generate a PWM signal. ATmega32u4 can receive values from sensors with analog signals via 12 ADC channels.

The "control" microcontroller is Qualcomm Atheros AR9331, which is built on the MIPS architecture. Its tasks include interaction with other LAN nodes. It operates at $400 \mathrm{MHz}$ and has $16 \mathrm{MB}$ of program memory and $64 \mathrm{MB}$ of RAM. The microcontroller runs on a special version of the OpenWRT operating system, which is based on the Linux kernel. The communication between the two microcontrollers takes place via the UART serial bus and the Bridge library.

Thus, Arduino Yun allows, on the one hand, to access the ZeroMQ library and, on the other hand, to generate PWM signals by hardware, as well as to work with a large number of analog sensors.

\subsection{Yun Shield}

This module is an expansion board for Arduino, developed by Dragino [10]. It is built using the Dragino HE board (in chinese "HE" stand for "core") running on the Atheros AR9331 microcontroller. The module has the same amount of memory and methods of interaction with Arduino as Arduino Yun.

This module creates additional opportunities for the use: since OpenWrt is a full - fledged Linux operating system, it is possible to collect a set of compilers for AVR microcontrollers and a set of tools to work with Arduino, which makes it possible to collect and update the software for the Arduino directly to the control system (CS), dynamically reprogramming the Executive Arduino MCP.

\subsection{Seeduino Cloud}

MCP [11] is a cheaper alternative to Arduino Yun, built using "HE” module.

\subsection{Intel Galileo}

It is a microcomputer that is compatible with Arduino [12]. It is built on a system-on-chip (SoC) Intel Quark x1000 with $\mathrm{x} 86$ architecture and a clock speed of $400 \mathrm{MHz}$. The board has 14 GPIO, of which 6 can generate PWM signal. The board has a six-channel ADC. Also on the board are: microSD memory card slot, connector for mini PCIe expansion cards and 2 microUSB connectors (one works in the "host" mode, the other - in the "guest" mode).

Of all the above MCPs, this one consumes the most energy (supply voltage $5 \mathrm{~V}$ at a current consumption of $3 \mathrm{~A}$ in peak modes). At the same time the main processor of MCP emits a lot of thermal energy which needs to be taken away with use of radiators and fans that negatively affects applicability of MCP in mobile robotics. Intel Galileo runs an operating system based on the Linux kernel.

However to install additional libraries and programs, you must install an operating system image on the plug-in memory card and configure it on the device. Intel Galileo does not need additional modules and is a ready-made solution for creating a control system.

\subsection{Raspberry Pi B+}

This microcomputer was created to teach programming and is used in schools in England [13]. MCP is based on the Broadcom BCM2835 system-on-chip (SoC). In turn, this SoC is based on the ARM processor ARM1176JZ F running at $700 \mathrm{MHz}$. The processor frequency can be programmatically increased to $1 \mathrm{GHz}$. The processor is connected to the RAM size of $512 \mathrm{MB}$, operating at a frequency of $400 \mathrm{MHz}$. On the board itself there is no permanent memory; the bootloader, operating system and other files are stored on a microSD memory card, the connector for which is located on the MCP. To work with external devices Raspberry Pi B+ is equipped with 4 USB connectors and an Ethernet connector. HDMI, RCA and $3.5 \mathrm{~mm}$ JACK connectors are available for audio and image output. On the board there are mounts for cables of standard camera and display.

There is also a two-row connector with GPIOs, some of which have a special purpose. The GPIO lines on the Raspberry Pi B+ have 3.3 V voltage levels, which does not allow direct connection of various devices with 5V signal levels. To work with such devices it is necessary to use logic level Converter. MCP Raspberry Pi B+ itself can generate complex signals, loading $100 \%$ of the main processor of the device. Also Raspberry Pi is not equipped with ADC, which, in turn, does not allow reading analog sensors. To unload the processor and to work with analog sensors, it is possible to use other MCP, such as Arduino Uno or Arduino Mega. Raspberry Pi B+ is capable of running AVR toolchain and programmer for MCP of different models. Thus, the Raspberry Pi B+ MCP implements dynamic reprogramming of all parts of the control system. 


\subsection{Orange Pi Zero}

The microcomputer is based on the Allwinner H2+ system-on-chip (SoC) based on the ARM Cortex-A7 Quad-core processor and the Mali400 MP2 graphics coprocessor [14]. This MCP operates at frequencies comparable to and even superior to the Raspberry Pi B+. Orange Pi Zero is equipped with 256 or $512 \mathrm{MB}$ of RAM. As a permanent storage device uses a removable MicroSD memory card or a memory chip soldered to the board. In addition, an AR100 microcontroller based on the OpenRISC1000 free core is built into the SoC, which is used to control the SoC in deep sleep mode. It can control all internal modules of the SoC, including GPIO.

The microcontroller AR100 mostly inactive or isn't used depending on OS. Hence, it can play the role of "Executive microcontroller" like ATMega microcontroller in Arduino Yun MCP. The interaction between the ARM processor and the built-in microcontroller occurs through internal memory, which is much more efficient than the UART bus used in Arduino Yun and does not require text serialization and deserialization of data.

It is worth noting the high learning curve for programming this microcontroller. But besides AR100, SoC has 4 main processors with hardware floating point math, which allows implementing a control system of almost any complexity. The capabilities of this MCP will be enhanced if it is possible to use the Linux kernel with real-time extensions.

\subsection{6duino ZERO plus}

MCP, similar to the Intel Galileo is compatible with Arduino software [15]. The microcomputer is built on the system-on-module (SoM) Vortex86EX SOM-128-EX. The 32 bit x86 architecture processor of the SoM operates at a frequency of $300 \mathrm{MHz}$, has $1 \mathrm{~GB}$ of RAM and $8 \mathrm{MB}$ of built-in flash memory, interfaces for connecting external storage devices (microSD) and peripheral devices on the PCI bus. It contains built-in interfaces for motion control (7 channels of 32-bit PWM and 1 counter channel for encoder with A, B and Z signal channels). Also on the board directly displayed interface PCI-e.

USB Host and Ethernet interfaces for minimization of the board area and compliance with the Arduino Leonardo board form factor are output to non-standard pin connectors. Adapters to standard Ethernet and USB connectors are produced and sold separately. This approach has its advantages in a modular robot - internal connections in module become less bulky.

Since the MCP 86duino ZERO plus has all the elements of a conventional IBM compatible computer, it is possible to directly install or relatively easy port any compatible OS, including most state-of-art real-time OS. Combined with hardware-based motion control, this allows for high-quality embedded systems. In addition to the listed interfaces, 86duino ZERO plus has RS-485 and CAN interfaces, which allows the use of modules from other modular robots (such as those discussed earlier in [1], [3]: Thor and SMART) or separate standard components from third-party manufacturers, such as servo motors or even cars that have CAN bus control.

\subsection{6duino ONE plus}

MCP, as well as the 86duino ZERO plus, is built on the same system-on-module and is, in fact, it's an extended version [16]. It has a bigger variety of different interfaces. For example, on the back of the board there is a connector for PCI-E modules, such as Wi-Fi module or VGA video card module. MCP outputs all SoM interfaces, including hardware generation of PWM and reading of external devices.

\subsection{Onion Omega2+ с платой Arduino Dock R2}

MCP Onion Omega2+ [17] as well as Arduino Yun, is based on MIPS-processor and runs Linux kernel based OS, but has a more productive core MediaTek MT7688 (clock speed-580 MHz, $128 \mathrm{MB}$ of RAM and $32 \mathrm{MB}$ of program memory). Also directly on the module there is a connector for MicroSD cards, which can further increase the amount of program memory up to several gigabytes.

In combination with the Arduino Dock R2 [18], MCP becomes an improved version of the Arduino Uno and Yun Shield combination due to the fact that the Arduino Dock R2, unlike the Yun Shield, has pins both from the Onion Omega2+ and Arduino-compatible connector. The drawback of Arduino Dock R2 is the lack of an Ethernet connector along with the necessary passive elements for its operation.

\section{Choosing module's computation platform for mechatronic device.}

Since many of the qualitative characteristics of the listed MCP often conflict each other, it is difficult to objectively distinguish any universal convolutional criteria for them. Therefore, it makes sense to apply multi-criteria Pareto optimization [19], [20]. Unlike conventional optimization with additive or multiplicative criteria, it is impossible to obtain a single optimal solution. Pareto optimization will allow one to select those MCPs that are closest to the solution of a specific problem. In the worst case this optimization will not shrink the search space at all. If this happens, one should revise number and meaning of quality criteria. 
It is proposed to divide all quality criteria of an MCP into several classes:

- Objective quality criteria (OQC) are the characteristics of MCP, which can be compared based on its datasheet.

- Computationally objective quality criteria (COQC) are those characteristics of the MCP that can be calculated by any known algorithm based on its datasheet.

- Subjective quality criteria (SQC) are those characteristics of MCP, which have an algorithm of calculation which is impossible to define formally. The values of these criteria are obtained by expert evaluation by designers of modules in the context of module being developed.

As an example of stated earlier, the following are the criteria used to assess the quality of the MCP to develop the transport module of a mobile robot with modular architecture They are mostly applicable in case of many other modules [1], [2], [3]:

- SQC1: the possibility of implementing the specifications of inter-module interaction [2]. The criterion is the sum of the sub-criteria:

- SQC1.1: an implementation of the ZeroMQ library for MCP (0 - implementations do not exist; 0,5 implementation is not completed, there are serious flaws; 1 - the implementation of the stable).

- SQC1.2: there are restrictions on the number of simultaneous connections to the interface $(-1-$ there are restrictions, and they are significant; 0 - there is no information about the restrictions; $1-$ there are no restrictions).

- SQC1.3: the Existence of restrictions on the number of simultaneous interfaces $(-1-$ limitations are there and they are significant; 0 -information no limitations; 1 - no limit).

- SQC2: the ability to create applications running in hard real time; the criterion is the sum of the sub-criteria:

- SQC2.1: real - time operating system Availability (0.5 - OS exists, but difficult to install/uninstall, 1-OS exists and easy to install and use).

- SQC2.2: the entry Level in programming in real time (-1 - high; 0 - average; 1 - poor).

- COQC3: MCP performance with floating numbers. It is estimated by running a special benchmark in the microcontroller emulator [4], which performs a lot of floating point calculations and reports the accuracy them. The processor time spent in the benchmark $(1 \mathrm{~ms}, 1 \mathrm{sec}, \ldots)$ is calculated based on the results of the launch.

- OQC4: Number of GPIO $(0,1,2, \ldots)$.

- OQC5: Bit width of the timers used in PWM generation $(0,1,2, \ldots$; usually $8,16,32)$.

- OQC6: Number of PWM channels $(0,1,2, \ldots)$.

- OQC7: Number of interrupt channels $(0,1,2, \ldots)$.

- COQC8: Number of full channels for working with encoders $(0,1,2, \ldots)$.

- OQC9: Number of ADC channels $(0,1,2, \ldots)$.

- SQC10: Cost of MCP; if MCP consists of several parts, its costs add up. Since the cost of MCP must be minimized, this criterion is multiplied by -1 .

- SQC11: Estimate time of delivery MCP in days. Like cost, delivery time must be minimized, so the criterion is multiplied by -1 .

\section{Optimization results}

Comparative analysis showed that 7 of the investigated MCPs are optimal:

- Arduino Uno + Ethernet Shield (SQC1=-1.5; SQC2=2; COQC3=0; OQC4=10; OQC5=8; OQC6=6; OQC7=2; OQC8=1; OQC9=6; SQC10=-2347; SQC11=-2347),

- Arduino Mega + Ethernet Shield (SQC1=-1.5; SQC2=2; COQC3=0; OQC4=50; OQC5=8; OQC6=15; OQC7=6; OQC8=3; OQC9=16; SQC10=-3547; SQC11=-3547),

- Taijiuino Due Pro R3 (SQC1=0.5; SQC2=1; COQC3=0,5; OQC4=54; OQC5=12; OQC6=12; OQC7=54; OQC8=27; OQC9=12; SQC10=-2699; SQC11=-2699),

- Seeeduino Cloud (SQC1=3; SQC2=2; COQC3=0; OQC4=18; OQC5=8; OQC6=7; OQC7=2; OQC8=1; OQC9=12; SQC10=-2850; SQC11=-2850),

- 86duino ZERO (K1=3; SQC2=1; COQC3=1; OQC4=14; OQC5=32; OQC6=7; OQC7=14; OQC8=4; OQC9=6; SQC10=-3690; SQC11=-3641),

- 86duino One (SQC1=3; SQC2=1; COQC3=1; OQC4=45; OQC5=32; OQC6=11; OQC7=45; OQC8=4; OQC9=7; $\mathrm{SQC10}=-5250 ; \mathrm{SQC11}=-5611)$

- Raspberry $\mathrm{Pi}$ B+ (SQC1=3; SQC2=-0,5; COQC3=1; OQC4=27; OQC5=0; OQC6=0; OQC7=0; OQC8=0; OQC9=0; SQC10=-3100; SQC11=-3100). 


\section{Experimental results}

At the present time a transport module and the whole mobile robot with modular architecture has different design and structure compared with results that were published in the paper [2]. It was decided to prototype the control system using module's computational platform consisting of Arduino Mega and Ethernet Shield for the new transport module design. The transport module consists of the following main components: two geared DC motors with Hall sensors, motor driver L298N and DC-DC converter for module's power supply. Figure 1 depicts laboratory prototype of the transport module and the prototype of transport module with power supply module.

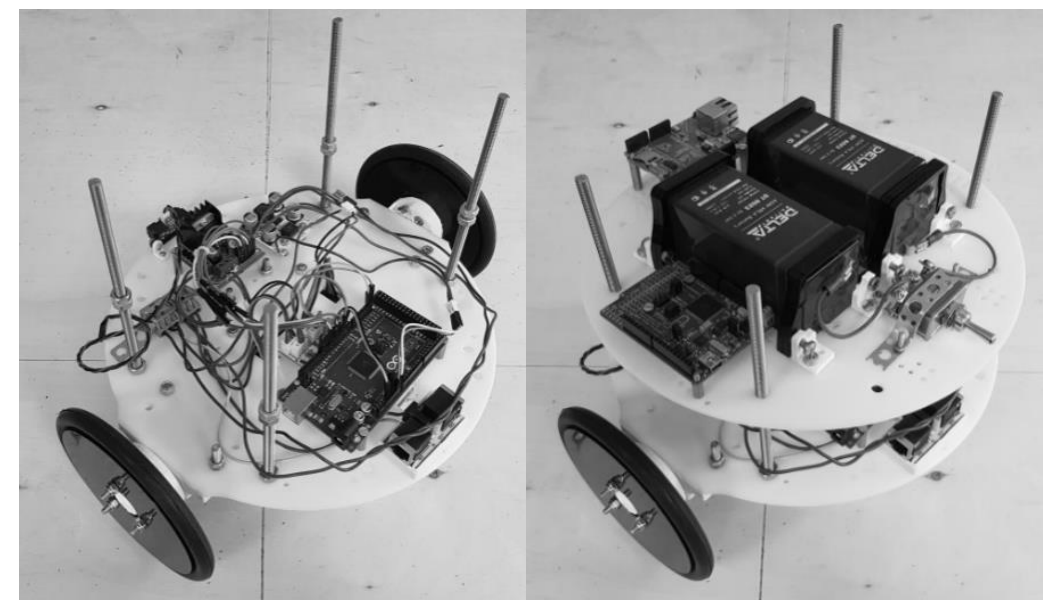

Fig. 1. Laboratory prototype of transport module (left); The transport module and power supply module

As long as all modules have similar flat design mechanical connection is performed by four threaded rods.

At this time control system is completely based on computing platform Arduino Mega that is responsible for motion planning and control of actuators. For wheels speed control integer PID controllers are used; it was necessary for real time computation of Arduino Mega. Mobile robot position is estimated using odometry. Several experiments has been performed for robot position accuracy analysis: movement to circle and its following, motion along straight line to the defined distance and motion nearby control points. The last experiment's result is shown on figure 2 . This figure shows mobile robot's trajectory based on odometry compared with trajectories derived from computer simulation. In this experiment robot was meant to go nearby three points with next coordinates: $(0.5,0),(0.5,0.25)$ и $(1,0.25)$. The robot's initial position is $(0,0)$.
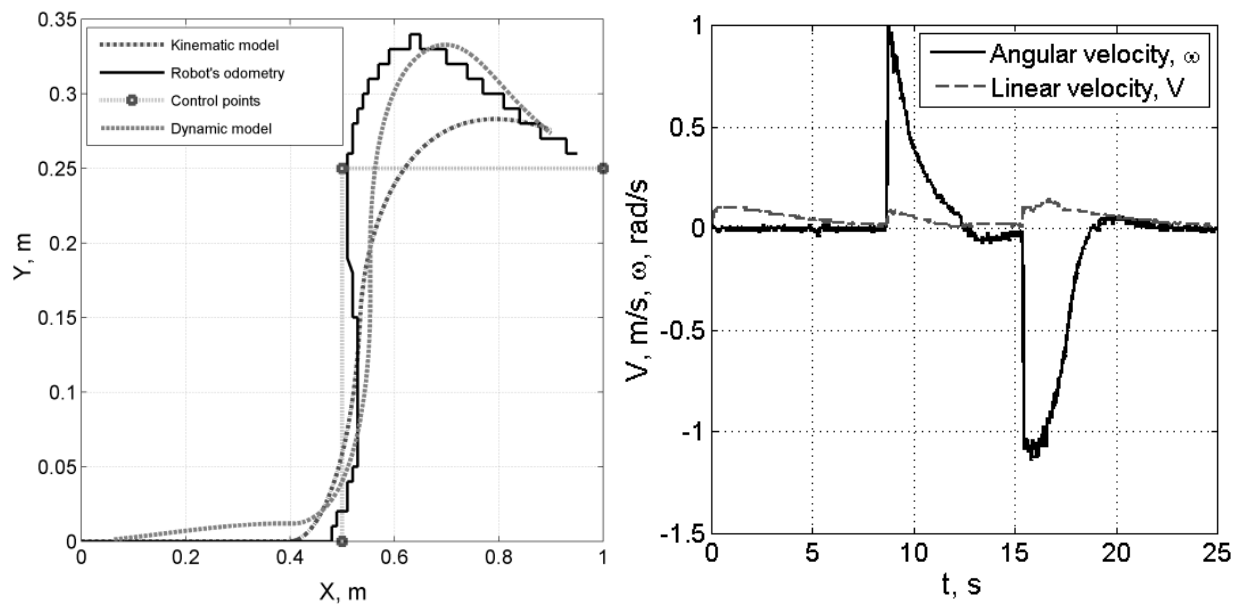

Fig. 2. Left: odometry based robot's trace compared with kinematic and dynamic models traces; right: robot's linear and angular velocities during the motion

Also figure 2 shows robot's linear and angular velocities during motion execution. One can see that maximum deviation between odometry based robot trajectory and robot's dynamic model trajectory derived from computer simulation doesn't exceed $60 \mathrm{~mm}$. This and other experiments show that module's odometry ensure necessary position accuracy at the small distances. In summary using module's computation platform that was chosen from the list provided by Pareto optimum allows to design working functional module if one applies specific methods for computation acceleration of the platform. 


\section{Conclusion}

The paper makes use of existing well-known method of multi-criteria optimization to narrow the problem space in choosing module's computational platform (MCP) for mobile robot with modular architecture. 15 MCPs and their sets were analyzed and evaluated using 11 quality criteria, some of which are generic (cost, GPIO count, etc) and some of which are specific for modular approach, introduced in previous works [1], [2], [3], [21] (networking abilities and realtime applicability). $7 \mathrm{MCPs}$ were proposed to be the fittest solutions for a control system of a module. Further experimentation using work from [3] and [21] and this paper showed applicability of presented results.

In the future it is possible to design other modules and test this technique of narrowing down search space with more concise criteria for each new module. Each day a new microcontroller or a new development and prototyping board is being developed, so this analysis could be widen each day; new microcontroller can bring new and powerful hardware and software to it.

\section{Acknowledgments}

The authors are expressing their sincere gratitude to S. Kuvshinov, Director of International Institute of the New Educational Technologies of Russian State University for the Humanities, for assistance in construction of the experimental equipment. This work was supported by the Russian Foundation for Basic Research, grants no. 16-0700811a \& 16-07-01264a.

\section{References}

[1] Andreev, V.; Kim, V. \& Pletenev, P. (2017). The principle of full functionality - the basis for rapid reconfiguration in heterogeneous modular mobile robots // Proceedings of the 28th DAAAM International Symposium, pp. 0023-0028, B. Katalinic (Ed.), Published by DAAAM International, ISBN 978-3-902734-11-2, ISSN 1726-9679, Vienna, Austria. DOI:10.2507/28th.daaam.proceedings.003.

[2] Andreev, V. \& Pletenev, P. (2017). Organizing Intermodular Communication for Heterogeneous Modular Mobile Robot, Proceedings of the 28th DAAAM International Symposium, pp.0474-0480, B. Katalinic (Ed.), Published by DAAAM International, ISBN 978-3-902734-11-2, ISSN 1726-9679, Vienna, Austria DOI: 10.2507/28th.daaam.proceedings.066

[3] Andreev, V. \& Kim, V. (2017). The application of artificial vector fields for motion control of a heterogeneous modular mobile robot, Proceedings of the 28th DAAAM International Symposium, pp. 0635-0644, B. Katalinic (Ed.), Published by DAAAM International, ISBN 978-3-902734-11-2, ISSN 1726-9679, Vienna, Austria. DOI: 10.2507/28th.daaam.proceedings.090.

[4] https://www.arduino.cc/en/main/arduinoBoardUno, (2017). Arduino Uno, Accessed on: 2017-03-17

[5] https://www.arduino.cc/en/Main/ArduinoEthernetShieldV1, (2017). Ethernet Shield, Accessed on: 2017-03-17

[6] https://www.arduino.cc/en/main/arduinoBoardMega2560, (2017). Arduino Mega 2560, Accessed on: 2017-03-17

[7] https://www.arduino.cc/en/main/arduinoBoardDue, (2017). Arduino Due, Accessed on: 2017-03-17

[8] http://www.emartee.com/product/42271/Taijiuino Due Pro R3 Arduino Due Compatible, (2017). Taijiuino Due Pro R3 + Ethernet PHY, Accessed on: 2017-03-17

[9] https://www.arduino.cc/en/main/arduinoBoardYun, (2017). Arduino Yun, Accessed on: 2017-03-17

[10] http://www.dragino.com/products/yunshield/item/86-yun-shield.html, (2017). Yun Shield, Accessed on: 2017-0317

[11] http://wiki.seeed.cc/Seeeduino_Cloud/, (2017). Seeeduino Cloud, Accessed on: 2017-03-17

[12] http://amperka.ru/product/intel-galileo, (2017). Arduino Galileo // Amperka (Амперка)., Accessed on: 2017-05-10

[13] http://amperka.ru/product/raspberry-pi-model-b-plus, (2017). Raspberry Pi Model B+ // Аmреrka (Амперка)., Accessed on: 2017-05-10

[14] http://www.orangepi.org/orangepizero/ (2017), orange pi zero - Orangepi Accessed on: 2017-03-17

[15] http://shop.dmp.com.tw/INT/products/155, (2017). 86Duino ZERO Plus, Accessed on: 2017-05-10

[16] http://shop.dmp.com.tw/INT/products/154, (2017). 86Duino ONE Plus, Accessed on: 2017-05-10

[17] https://docs.onion.io/omega2-docs/omega2p.html, (2017). Onion Omega2+ // Onion Omega2 Documentation, Accessed on: 2017-05-10

[18] https://docs.onion.io/omega2-docs/arduino-dock-2.html, (2017). Arduino Dock 2 // Onion Omega2 Documentation, Accessed on: 2017-05-10

[19] Lukinov, A.P. (2012). Design of mechatronic and robotic devices, (Лукинов А.П. Проектирование мехатронных и робототехнических устройств), Lan(Лань), ISBN: 978-5-8114-1166-5, Moscow, Russia.

[19] http://systematy.ru/articles/44_optimizatsiya_vektornogo_kriteriya_pareto-optimalnyie_resheniya, (2017). Glukhikx I.N. Optimization of a vector criteria. Pareto-optimal solutions. (Глухих И.Н. Оптимизация векторного критерия. Парето-оптимальные решения), Accessed on: 2017-03-17

[20] Walker, J (2016). Floating Point Benchmarks, Available from: https://www.fourmilab.ch/fbench/, Accessed on: 2018-09-01

[21] Andreev, V \& Kim, V. (2016). Control system and design of the motion module of a heterogeneous modular mobile robot, Proceedings of the 27th DAAAM International Symposium, pp. 586-594, B. Katalinic (Ed.), Published by DAAAM International, ISBN 978-3-902734-08-2, ISSN 1726-9679, Vienna, Austria, DOI: 10.2507/27th.daaam.proceedings.086. 